\title{
Criminologie
}

\section{Quand vieillir ensemble fait mal : les mauvais traitements entre conjoints âgés}

\section{Sylvie Gravel, Marie Beaulieu et Maxine Lithwick}

Volume 30, numéro 2, automne 1997

Violences familiales

URI : https://id.erudit.org/iderudit/017405ar

DOI : https://doi.org/10.7202/017405ar

Aller au sommaire du numéro

\section{Éditeur(s)}

Les Presses de l'Université de Montréal

ISSN

0316-0041 (imprimé)

1492-1367 (numérique)

Découvrir la revue

Citer cet article

Gravel, S., Beaulieu, M. \& Lithwick, M. (1997). Quand vieillir ensemble fait mal : les mauvais traitements entre conjoints âgés. Criminologie, 30(2), 67-85.

https://doi.org/10.7202/017405ar
Résumé de l'article

This article focuses on a rarely discussed and relatively unknown type of elder abuse: elder abuse by a spouse. The data presented comes from a study examining the dynamics of elder abuse cases in three CISC's in Quebec (Local Centre for Community Services). Firstly, the characteristics associated with forms of abuse and both the abused spouse and the abusive spouse are described. Cases of elder abuse by a spouse are also compared with the other cases of elder abuse in the sample: abuse by a child, another family member, a friend or an acquaintance. Secondly, the authors examine more specifics situations of elder abuse by a spouse such as situations where one of the spouses has cognitive impairment and how that differs from situations where both spouses are lucid. For each of these situations, the different elements having an impact on the dynamics of abuse are discussed. 


\section{QUAND VIEILLIR ENSEMBLE FAIT MAL : LES MAUVAIS TRAITEMENTS ENTRE CONJOINTS ${ }^{1}$ ÂGÉS Sylvie Gravel ${ }^{2}$, Marie Beaulieu ${ }^{3}$ et Maxine Lithwick ${ }^{4}$}

This article focuses on a rarely discussed and relatively unknown type of elder abuse: elder abuse by a spouse. The data presented comes from a study examining the dynamics of elder abuse cases in three CLSC's in Quebec (Local Centre for Community Services). Firstly, the characteristics associated with forms of abuse and both the abused spouse and the abusive spouse are described. Cases of elder abuse by a spouse are also compared with the other cases of elder abuse in the sample: abuse by a child, another family member, a friend or an acquaintance. Secondly, the authors examine more specifics situations of elder abuse by a spouse such as situations where one of the spouses has cognitive impairment and how that differs from situations where both spouses are lucid. For each of these situations, the different elements having an impact on the dynamics of abuse are discussed.

Le contenu du présent article est tiré d'une recherche-action menée en partenariat avec trois CLSC et subventionnée par le Conseil québécois de recherche sociale (CQRS). L'étude visait à mieux comprendre les différentes dynamiques associées aux mauvais traitements à l'égard des personnes âgées, de manière à développer dans les CLSC des stratégies d'intervention plus efficaces.

Dans le cadre de cet article, nous abordons une dimension spécifique des mauvais traitements à l'égard des personnes âgées, celle des mauvais traitements entre conjoints âgés. Après une brève mise en contexte et la masculin.

1. Afin d'alléger la lecture du texte, le terme « conjoint » englobe le genre féminin et

2. M.Sc., professionnelle en recherche, Université de Montréal, C.P. 6128, succursale Centre-Ville, Montréal, QC, H3C 3J7.Adresse électronique : gravels@magellan.umontreal.ca

3. Ph.D., professeure au Département des sciences humaines de l'Université du Québec à Rimouski, 300, allé des Ursulines, Rimouski, QC, G5L 3A1.

4. M.Sw., coordonnatrice des Services en gérontologie sociale au CLSC René-Cassin/Institut universitaire de gérontologie sociale du Québec, 5800 boul. Cavendish, suite 600, Côte St-Luc, QC, H4W 2T5.

5. Les termes « personne âgée » et « aîné » englobent toute personne âgée de 60 ans et plus. 
présentation de l'approche méthodologique utilisée, nous faisons la description des situations de mauvais traitements infligés par un conjoint âgé : les formes de mauvais traitements et les caractéristiques sociodémographiques des conjoints victimes et des conjoints auteurs de mauvais traitements sont décrites et comparées au reste de l'échantillon, c'est-à-dire aux situations où celui qui inflige les mauvais traitements est un enfant de la personne âgée, un autre membre de la famille (bru, neveu, etc.), un ami ou une connaissance.

L'étude a identifié un certain nombre de situations de mauvais traitements comprenant un conjoint souffrant de pertes cognitives. Afin de mieux comprendre les dynamiques qui sous-tendent les mauvais traitements au sein des couples âgés, nous avons cru bon de séparer, aux fins de l'analyse, les couples dont les deux conjoints sont lucides de ceux dont l'un des membres souffre de pertes cognitives. Dans la deuxième partie, les situations plus spécifiques de mauvais traitements entre conjoints lucides sont donc abordees. Enfin, l'article se termine par l'analyse des situations où l'un des conjoints souffre de pertes cognitives. Dans chacune de ces deux dernières sections, les différents éléments jouant un rôle dans la dynamique des mauvais traitements sont discutés.

\section{MISE EN CONTEXTE}

C'est au tournant des années 80 qu'apparaît le concept de mauvais traitements à l'égard des personnes âgées. Depuis, un nombre relativement important de recherches ont été réalisées sur le sujet, dont la majorité proviennent des États-Unis. Les données recueillies divergent d'une étude à l'autre en raison des lacunes méthodologiques de la plupart d'entre elles et des divergences qui existent au sujet de la définition des mauvais traitements. Malgré cela, d'un grand nombre de ces études ressort le portrait suivant de la personne âgée maltraitée : il s'agit d'une femme, assez âgée (75 ans en moyenne), dépendante des membres de sa famille sur le plan physique mais aussi psychologique et financier (Lévesque, 1990). Le profil de la personne qui maltraite est tout aussi uniforme : il s'agit d'un membre de la famille (le plus souvent un enfant), qui doit prendre soin de son parent en perte d'autonomie physique, et qui, mal préparé à sa tâche d'aidant, subit des tensions importantes dues à cette nouvelle situation, ce qui le mène à maltraiter et négliger son parent âgé (Lévesque, 1990 ; Namiash, 1990).

Par contre, quelques études tracent un portrait de la personne âgée maltraitée qui tranche avec les résultats des autres recherches. Entre autres, les résultats obtenus par Pillemer et Finkelhor (1988) indiquent que les hommes sont plus fréquemment victimes de mauvais traitements que les 
femmes, que la dépendance physique n'est pas un trait spécifique aux personnes âgées maltraitées, et que la majorité de ces dernières ne sont pas maltraitées par un de leurs enfants mais plutôt par leur conjoint. Leurs données proviennent d'un sondage réalisé auprès d'un échantillon représentatif de la population, ce qui exclut les biais propres aux échantillons construits à partir des cas recensés dans des organismes de santé ou de services sociaux.

D'autres études (Shell, 1982 ; Giordano et Giordano, 1984 ; Wolf $e t$ al., 1984 ; Grandmaison, 1988) appuyaient l'hypothèse que les mauvais traitements entre conjoints âgés ne constituent pas un phénomène marginal. Dans le sillage de ces études, un nouveau concept est apparu, celui du «spouse abuse grows old».

Ces études n'ont guère eu d'impact sur les chercheurs et les praticiens. On constate qu'on a peu fait pour tenter de comprendre cette problématique spécifique des mauvais traitements entre conjoints âgés. Toutefois, la publication de quelques études récentes (Suitor et al., 1990 ; Vinton, 1992 ; Aronson et al., 1995 ; Harris, 1996) indiquent que les mauvais traitements au sein des couples âgés commencent à susciter un plus grand intérêt de la part des chercheurs. Soulignons, par ailleurs, que les chercheurs en violence conjugale se sont jusqu'ici très peu intéressés à la question spécifique des femmes âgées violentées par leur conjoint.

La plupart des études dans le domaine des mauvais traitements envers les aînés ont envisagé les mauvais traitements dans la perspective d'une personne âgée physiquement dépendante (victime potentielle de mauvais traitements) et d'un aidant naturel qui lui prodigue des soins quotidiens et qui la maintient dans son milieu naturel (abuseur potentiel). Cette façon de poser le problème a eu pour conséquence d'occulter certaines dimensions du phénomène des mauvais traitements à l'égard des aînés. Ainsi, les chercheurs ont très peu discuté des mauvais traitements infligés par des individus qui ne jouent pas le rôle d'aidant auprès de la personne âgée ainsi que des mauvais traitements dont sont victimes des personnes âgées totalement autonomes physiquement.

Notre démarche de recherche, essentiellement heuristique, $s^{\prime}$ inscrit dans une volonté d'appréhender les mauvais traitements à l'égard des personnes âgées comme un phénomène multidimensionnel, afin d'identifier les différences qui existent entre les diverses situations de mauvais traitements et, ultimement, d'élaborer des outils d'intervention plus efficaces. C'est dans cette optique que nous avons choisi d'examiner les mauvais traitements et les dynamiques qui les sous-tendent sous l'angle du lien spécifique unissant la personne âgée à la personne qui la maltraite. Nous avons donc étudié les mauvais traitements selon qu'ils sont infligés par un conjoint, un enfant, ou 
une connaissance ${ }^{6}$. Dans le cadre de cet article, c'est plus spécifiquement des mauvais traitements entre conjoints âgés qu'il sera question.

\section{QUELQUES INFORMATIONS MÉTHODOLOGIQUES}

De la littérature sur les mauvais traitements envers les aînés, un constat émerge : il n'y a toujours pas de consensus entre les chercheurs au sujet d'une définition des mauvais traitements (Valentine et Cash, 1986; Finkelhor et Pillemer, 1988 ; McDonald et al., 1991 ; Maclean, 1995). Dans le cadre de cette étude, nous avons retenu les quatre formes de mauvais traitements autour desquelles se cristallise la plus large adhésion : les mauvais traitements physiques, les mauvais traitements psychologiques, l'exploitation financière et la négligence, tant active que passive. Ces différentes formes de mauvais traitements regroupent les réalités suivantes :

1. Mauvais traitements physiques : agression sexuelle, agression physique telle que poussées, contentions, coups, étranglement, etc.

2. Mauvais traitements psychologiques : violence verbale, intimidation, menaces, harcèlement, contrôle de la personne âgée, refus de la faire participer aux prises de décision la concernant, chantage affectif, blâme, culpabilisation, indifférence.

3. Exploitation financière : réclamations frauduleuses, vivre aux crochets de la personne âgée, réclamations continuelles d'argent sous toutes sortes de prétextes, compte de banque "vidé " par un individu à qui la personne âgée a signé une procuration, vol d'argent ou de certains biens.

4. Négligence : situations où les besoins primaires et secondaires de la personne âgée ne sont pas satisfaits : hygiène inappropriée, nourriture insuffisante ou inadéquate, soins médicaux inadéquats, mauvaises conditions de vie, vêtements insuffisants, absence de prothèses, supervision inadéquate. La négligence active consiste en un refus délibéré de répondre aux besoins de la personne âgée. La négligence passive consiste en un refus non délibéré, dû à un manque de connaissances, de répondre aux besoins de la personne âgée.

L'étude porte sur les situations de mauvais traitements qui affectent les personnes âgées vivant dans la collectivité, c'est-à-dire qui ne sont pas hébergées en institution privée ou publique. Nous avons adopté une définition large des mauvais traitements englobant tous les mauvais traitements

6. La catégorie « connaissance » inclut aussi d'autres membres de la famille (bru, neveu, etc.). 
infligés à une personne âgée, quel que soit le lien entre cette dernière et l'auteur des mauvais traitements, à l'exception des actes commis par des inconnus, lesquels relèvent sans aucun doute d'une problématique particulière.

En outre, la définition retenue inclut des actes qui ne sont pas nécessairement infligés d'une manière volontaire ou intentionnelle. Ainsi, l'étude comprend des situations où le conjoint qui maltraite souffre de pertes cognitives. Par pertes cognitives on entend les pertes de mémoire sévères, la démence et la maladie d'Alzheimer. Bien qu'ayant de grandes réticences à qualifier ces personnes « auteurs de mauvais traitements », parce que, d'une part, le terme possède bien sûr un sens péjoratif, et que, d'autre part, dans bien des cas, il semble évident que leur comportement est une conséquence de leur état mental, nous avons choisi d'éviter d'émettre des jugements de valeur, à l'égard de la personne qui commet les mauvais traitements comme à celle qui les subit. Par ailleurs, dans une perspective de compréhension des dynamiques qui sous-tendent les différentes manifestations de mauvais traitements, il nous est apparu d'autant plus pertinent d'inclure les situations où le conjoint qui maltraite souffre de pertes cognitives que, comme nous le verrons plus loin, on retrouve dans plusieurs de ces situations des mauvais traitements réciproques, c'est-à-dire infligés par la personne atteinte de pertes cognitives envers son conjoint, et par le conjoint lucide envers son partenaire souffrant de pertes cognitives.

Les situations de mauvais traitements ont été identifiées par des intervenants sociaux de trois CLSC entre août 1994 et août 1995. Il s'agit des CLSC de l'Estuaire à Rimouski, Centre-Sud, situé dans le quartier du même nom à Montréal, et René-Cassin, implanté dans le secteur Côte St-Luc, Hampstead et Snowdon-ouest, dans l'Ouest de Montréal. Ces trois CLSC ont avant tout été choisis parce qu'ils desservent des populations comportant une importante proportion de personnes âgées. Puisqu'ils n'ont pas été sélectionnés au hasard, ils ne sont pas nécessairement représentatifs de l'ensemble des CLSC du Québec. Dans ces circonstances, il serait téméraire d' affirmer que les données de l'étude sont directement généralisables à l'ensemble du Québec.

Pour chaque situation de mauvais traitements identifiée, un questionnaire portant sur la victime des mauvais traitements, la personne infligeant les mauvais traitements, les divers aspects de la situation et les interventions effectuées a été complété par l'intervenant, à la suite de son évaluation de la situation. Nous avons également recueilli de l'information qualitative sur chaque situation de mauvais traitements sous la forme de questions ouvertes incluses dans le questionnaire, et d'une histoire de cas préparée par l'intervenant en charge du dossier. Au total, on a identifié 128 situations de mauvais 
traitements. De ces situations, 61 cas, soit près de la moitié, concernent des mauvais traitements entre conjoints âgés.

\section{LES SITUATIONS DE MAUVAIS TRAITEMENTS INFLIGÉS PAR UN CONJOINT ÂGÉ}

\subsection{Les mauvais traitements infligés}

La violence psychologique constitue la forme la plus commune de mauvais traitement infligé par un conjoint : elle se retrouve dans $86,9 \%$ des situations (tableau 1). Elle est beaucoup plus rare dans le reste de l'échantillon $(55,2 \%)$. Chez les couples étudiés, la forme de mauvais traitement psychologique la plus fréquente est, de loin, la violence verbale $(75,5 \%)$, c'est-à-dire les insultes, critiques, cris, dénigrements, humiliations, suivie par les manifestations de contrôle $(35,8 \%)$, c'est-à-dire le contrôle des allées et venues de la personne âgée ou de ses appels téléphoniques, les prises de décision unilatérales concernant le menu, l'heure du lever ou du coucher, etc. Dans le quart des situations de mauvais traitements psychologiques, la personne âgée est l'objet de menaces et d'intimidations : menaces de sévices physiques, de mort, gestes intimidants tels que des coups de poing sur la table.

\section{Tablean 1}

Les formes de mauvais traitements infligés au sein des couples âgés en comparaison avec le reste de l'échantillon

\begin{tabular}{|c|c|c|c|c|c|c|}
\hline \multirow[t]{2}{*}{ Formes de mauvais traitements ${ }^{7}$} & \multicolumn{2}{|c|}{ Couples âgés } & \multicolumn{2}{|c|}{$\begin{array}{c}\text { Reste de } \\
\text { l'échantillon }\end{array}$} & \multirow[b]{2}{*}{ Phi } & \multirow[b]{2}{*}{$p^{8}=$} \\
\hline & $\mathbf{n}$ & $\%$ & $\mathbf{n}$ & $\%$ & & \\
\hline Psychologiques & 53 & 86,9 & 37 & 55,2 & $-.34^{9}$ & .000 \\
\hline Physiques & 19 & 31,1 & 7 & 10,4 & -.25 & .004 \\
\hline Négligence & 14 & 23,0 & 27 & 40,3 & .19 & .03 \\
\hline Exploitation financière & 8 & 13,1 & 44 & 65,7 & .53 & .000 \\
\hline TOTAL DES SITUATIONS & 61 & & 67 & & & \\
\hline
\end{tabular}

7. Les catégories ne sont pas mutuellement exclusives.

8. Le seuil de signification acceptable a été fixé à .05 .

9. Il est à noter que dans le tableau 1, le coefficient Phi a été obtenu en opposant chacune des formes de mauvais traitement à l'ensemble des autres formes. Ainsi, le Phi .34 relié à la violence psychologique est le résultat de l'opposition entre les situations où il y a violence psychologique et les situations où il n'y a pas de violence psychologique. 
Les mauvais traitements physiques $(31,1 \%)$ sont également plus répandus chez les couples que dans le reste de l'échantillon $(10,4 \%)$. Au sein des couples âgés, la violence physique constitue la deuxième forme de mauvais traitement la plus courante, alors qu'elle est la forme la moins fréquente dans le reste de l'échantillon. La violence physique se traduit surtout par des coups, gifles, poussées et contentions.

La négligence se retrouve dans près du quart des situations. C'est un peu moins que dans le reste de l'échantillon où la négligence constitue 40,3\% des situations. La négligence prend la forme, dans $85,7 \%$ des cas, d'un manque de supervision du conjoint.

L'exploitation financière est, bien évidemment, beaucoup plus présente dans le reste de l'échantillon, où elle constitue d'ailleurs, avec une proportion de $65,7 \%$, la forme de mauvais traitement la plus fréquente. Chez les couples âgés, elle se traduit essentiellement par un contrôle financier exercé par le mari au détriment de sa conjointe.

En bref, nos données indiquent que la violence psychologique caractérise la grande majorité des situations impliquant des conjoints âgés, et que, dans un grand nombre de ces situations, elle n'est accompagnée d'aucune autre forme de mauvais traitement. En outre, la violence physique, qui est toujours accompagnée de violence psychologique, se retrouve dans une proportion non négligeable de situations, soit trois sur dix, constituant ainsi la deuxième forme de mauvais traitement la plus répandue.

Ces données, quant aux formes de mauvais traitements infligés par des conjoints âgés, correspondent à celles d'un sondage national effectué au Canada auprès de personnes âgées par Podnieks et Pillemer (1990). Leurs résultats indiquent que la majorité des auteurs de violence physique et verbale sont des conjoints. Une autre étude, effectuée aux États-Unis, montre également que la violence physique est plus fréquemment le fait d'un conjoint (Giordano, 1982).

\subsection{Caractéristiques des conjoints maltraités et des conjoints qui maltraitent}

Chez les couples âgés, comme dans le reste de l'échantillon, la majorité des victimes, soit environ trois victimes sur quatre, sont des femmes (tableau 2). En conséquence, puisque, à l'exception d'un couple lesbien, l'ensemble des autres couples est hétérosexuel, ce sont majoritairement les hommes qui maltraitent au sein des couples âgés. Seulement 18 personnes sur les 61 auteurs de mauvais traitements sont des femmes. 


\section{Tableau 2}

Caractéristiques des couples âgés où l'un des conjoints maltraite l'autre en comparaison avec le reste de l'échantillon

Victime des manvais traitements

\begin{tabular}{|c|c|c|c|c|}
\hline & $\begin{array}{l}\text { Couples âgés } \\
\qquad(\mathrm{n}=61)\end{array}$ & $\begin{array}{c}\text { Reste de } \\
\text { l'échantillon } \\
(n=66)\end{array}$ & & \\
\hline & $\%$ & $\%$ & Phi & $\mathrm{p}=$ \\
\hline Femume & 72,1 & 77,3 & n.s. & \\
\hline Soixantaine & 21,7 & 7,1 & -.17 & .05 \\
\hline Autonomie physique & 56,7 & 31.7 & -.25 & .005 \\
\hline Pertes cognitives & 24,6 & 37,9 & n.s. & \\
\hline Aidante & 39,3 & 1,5 & -.48 & .000 \\
\hline \multicolumn{5}{|c|}{ Auteur des mauvais traitements } \\
\hline & $\begin{array}{l}\text { Couples âgés } \\
\qquad(n=61)\end{array}$ & $\begin{array}{c}\text { Reste de } \\
\text { l'échantillon } \\
(n=66)\end{array}$ & & \\
\hline & $\%$ & $\%$ & Phi & $\mathbf{p}=$ \\
\hline Homme & 70,5 & 60,6 & n.s. & \\
\hline 60 ans et plus & 96,6 & 14,3 & -.81 & .000 \\
\hline Perte d'autonomie physique & 46,7 & 4,6 & -.48 & .000 \\
\hline Pertes cognitives & 24,6 & 0 & -.38 & .000 \\
\hline Aidant & 42,6 & 48,5 & n.s. & \\
\hline
\end{tabular}

Les victimes de mauvais traitements de la part de leur conjoint sont plus jeunes que celles du reste de l'échantillon ${ }^{10}$. Elles ont en moyenne 75,9 ans, comparativement à 78,8 ans pour le reste de l'échantillon. Si l'on examine plus attentivement les différences entre les catégories d'âges, on constate que chez les couples, il y a davantage de victimes dans la soixantaine et moins de victimes âgées de 80 ans et plus. Quant aux conjoints qui maltraitent, ils sont évidemment plus âgés que les auteurs de mauvais traitements du reste de l'échantillon : la presque totalité $(96,6 \%)$ est âgée de 60 ans et plus, alors que seulement $14,3 \%$ des auteurs du reste de l'échantillon sont dans la même catégorie d'âge.

Les personnes âgées victimes de leur conjoint sont plus autonomes que les victimes du reste de l'échantillon : $56,7 \%$ sont complètement autonomes

10. $($ T-value $=2,05 p=.04)$. 
pour les activités de la vie quotidienne ${ }^{1 \mathrm{l}}$, alors que c'est le cas de seulement $31,7 \%$ des victimes du reste de l'échantillon. Ces données, indiquant que près de six conjoints âgés maltraités sur dix sont autonomes physiquement, vont à l'encontre des résultats de plusieurs études suggérant que la perte d'autonomie physique constitue un important facteur de risque associé aux mauvais traitements (Block et Sinnott, 1979 ; Lau et Kosberg, 1979 ; Steinmetz et Amsden, 1983 ; Steinmetz, 1988).

Par ailleurs, nos données indiquent qu'une proportion importante de conjoints qui maltraitent, soit $46,7 \%$, n'est pas complètement autonome pour les activités de la vie quotidienne, alors que c'est le cas de seulement $4,6 \%$ des auteurs de mauvais traitements du reste de l'échantillon. Ce qui est très étonnant, c'est que les conjoints qui maltraitent sont plus nombreux (46,7\%) que les conjoints maltraités $(43,3 \%)$ à être dépendants physiquement. Encore une fois, ce résultat remet en cause l'idée de considérer la perte d'autonomie physique comme étant un des plus importants facteurs de risque associés aux mauvais traitements.

Le quart des conjoints maltraités et le quart des conjoints qui maltraitent souffrent de pertes cognitives. On constate une plus grande proportion de victimes atteintes de pertes cognitives dans le reste de l'échantillon $(37,9 \%)$, mais la différence observée n'est pas statistiquement significative.

Dans plus de huit situations sur dix, un des conjoints est l'aidant de l'autre. La littérature sur les mauvais traitements envers les personnes âgées a accordé, jusqu'à tout récemment, beaucoup d'importance aux situations où les mauvais traitements sont infligés par un aidant stressé et complètement débordé par la tâche à accomplir (Steinmetz et Amsden, 1983 ; Steinmetz, 1988). Or, si plus de quatre conjoints qui maltraitent sur dix agissent à titre d'aidant auprès de leur partenaire, nos données indiquent aussi qu'à peu près la même proportion des conjoints maltraités, soit $39,3 \%$, sont les aidants du partenaire qui les maltraite. Cette situation, où l'aidant est maltraité par la personne âgée dont il prend soin, a déjà été soulignée par Steinmetz (1988). Celle-ci fut l'une des premières à illustrer le fait que les mauvais traitements ne sont pas toujours à sens unique et que certaines personnes âgées font elles aussi usage de violence psychologique et physique envers les personnes qui s'occupent d'elles.

11. L'autonomie a êté mesurée par rapport à sept activités de la vie quotidienne : prendre son bain ou sa douche, manger, s'habiller, se déplacer à l'intérieur de la maison, se déplacer à l'extérieur de la maison, aller aux toilettes et prendre ses médicaments. 


\section{LES MAUVAIS TRAITEMENTS ENTRE CONJOINTS LUCIDES}

Dans notre échantillon, 32 couples sur 61 sont formés de conjoints ne souffrant pas de pertes cognitives. C'est plus précisément de ces couples dont il sera question dans cette section.

\subsection{Description des situations de mauvais traitements}

a) Les mauvais traitements infligés ${ }^{12}$

Les mauvais traitements infligés, encore une fois, sont très majoritairement de nature psychologique. On les retrouve dans 31 cas sur 32. Quant à la violence physique, elle est présente assez fréquemment, soit dans 12 situations sur 32. Enfin, la négligence n'a été observée que dans cinq dossiers sur 32.

b) Caractéristiques des victimes et des auteurs des mauvais traitements

Encore une fois, la majorité des victimes sont des femmes $(78,1 \%)$. On dénombre seulement sept femmes parmi les conjoints qui maltraitent. Mais trois d'entre elles sont à la fois auteures et victimes de mauvais traitements, ce qui signifie que les mauvais traitements sont réciproques entre les deux conjoints.

Dans les couples où les deux conjoints sont lucides, les partenaires victimes sont plus jeunes que dans les couples où l'un des partenaires souffre de pertes cognitives : les premiers ont en moyenne 73,1 ans alors que les seconds sont âgés en moyenne de 78,9 ans $^{13}$. On constate des résultats similaires chez les conjoints qui maltraitent : ceux provenant d'un couple ou les deux conjoints sont lucides sont plus nombreux à être âgés de 69 ans et moins $(33,4 \%)$ que les autres $(7,1 \%)^{14}$. Ces données peuvent s'expliquer simplement par le fait que les pertes cognitives sont associées positivement à l'âge de la personne : plus une personne est âgée, plus les probabilités qu'elle souffre de pertes cognitives augmentent.

Encore une fois, on remarque que les conjoints qui maltraitent sont plus nombreux à être dépendants physiquement que les conjoints maltraités. Ainsi, $32.3 \%$ des conjoints lucides victimes de mauvais traitements ne sont pas totalement autonomes pour les activités de la vie quotidienne, alors que c'est le cas pour près de la moitié des conjoints auteurs de mauvais traitements $(48,4 \%)$.

12. L'exploitation financière n'a pas été analysée parce qu'elle n'est présente que dans huit cas sur 61 .

13. $\mathrm{T}$-value $=2,80 \mathrm{p}=.007$.

14. $\mathrm{Phi}=.32 \mathrm{p}=.02$. 


\subsection{Dynamiques des mauvais traitements}

Nous avons voulu, dans cette section, identifier les éléments qui influent sur la dynamique des mauvais traitements entre conjoints âgés. Trois facteurs émergent de l'analyse. Il s'agit des antécédents de violence conjugale au sein du couple, de la perte d'autonomie du partenaire qui maltraite et de la perte d'autonomie du partenaire maltraité.

a) Une situation de violence conjugale qui a vieilli avec le couple

Une des questions qui surgit lorsqu'on aborde les mauvais traitements au sein du couple âgé est de savoir si ceux-ci sont apparus récemment ou s'ils sont l'expression d'une situation de violence conjugale qui a vieilli avec le couple. Lorsque les deux conjoints sont lucides, dans $68,8 \%$ des situations, les intervenants ont identifié que le couple vivait de la violence conjugale depuis de nombreuses années, parfois même depuis le début du mariage ${ }^{15}$. Plus précisément, dans plus des deux tiers des cas, la violence durait depuis plus de 40 ans.

Dans notre échantillon, à une exception près, c'est la femme qui est victime de cette violence. Dans certains cas (7/32), les deux conjoints la subissent et l'infligent à la fois. Dans tous les cas de violence conjugale récurrente que nous avons répertoriés, les mauvais traitements étaient de nature psychologique, et accompagnés dans la moitié des cas de violence physique.

Selon Gesino et al. (1982), qu'elles soient jeunes ou âgées, les femmes demeurent avec leur conjoint violent pour des motifs similaires : elles ont une piètre image d'elles-mêmes, elles sont isolées socialement, elles croient que le conjoint va changer, elles ressentent de la honte, elles ont peur de subir des représailles, elles ne veulent pas être stigmatisées par leur famille et leurs amis. Ces auteurs avancent que ces facteurs agissent plus fortement sur les femmes âgées en raison de leur socialisation plus traditionnelle et de l'importance qu'elles accordent à l'institution du mariage. Quant à Pagelow (1981), elle estime que la femme âgée, au contraire de ses consœurs plus jeunes, est souvent persuadée qu'il est trop tard pour modifier le cours des choses et recommencer une vie nouvelle.

Par ailleurs, nous croyons qu'il ne faut pas sous-estimer un aspect gérontologique important lorsqu'il s'agit de femmes âgées victimes de violence conjugale : la peur de vieillir seule. Notre étude n'a pas recueilli de données systématiques à ce sujet, mais nous posons l'hypothèse que la

15. Notons que dans $21,9 \%$ des situations, les intervenants ne possédaient pas assez d'informations leur permettant d'évaluer s'il s'agissait ou non de violence qui durait depuis plusieurs années. 
perspective de finir ses jours seule, avec tout ce que cela comporte d'incertitudes (Que va-t-il m'arriver? Qui va prendre soin de moi ? Vais-je devoir quitter mon logement, ma maison ?) peut constituer un frein supplémentaire à l'action. Le fait de vieillir avec son conjoint, sans bien sûr éliminer ces incertitudes, peut diminuer l'anxiété ressentie face à l'avenir. Ajoutons à cela que les femmes qui vivent dans un milieu de violence psychologique et physique depuis un grand nombre d'années peuvent avoir adopté, au fil du temps, des comportements d'acceptation et de résignation, et intégré un état de victime $^{16}$.

Quand on veut comprendre les mauvais traitements à l'intérieur des couples âgés, il faut remettre leur relation dans le contexte dans lequel elle est née et a évolué. N'oublions pas que les femmes qui sont aujourd'hui âgées se sont mariées dans une société plus sexiste et plus patriarcale que celle d'aujourd'hui, qui ne leur accordait que très peu de droits. Le seul modèle de fermme que la société valorisait à l'époque était celui d'épouse et de mère subordonnée à un mari, qui, lui, se voyait principalement relégué au rôle de pourvoyeur. La plupart des hornmes de cette génération ont exercé un contrôle important sur les décisions du ménage et même, dans plusieurs cas, sur la vie de leur conjointe. Rendus à un âge avancé, et même moins autonomes physiquement, ces hommes veulent continuer d'exercer leur pouvoir.

Il s'est avéré très difficile pour les intervenants de comprendre de quelle manière la violence dans le couple avait évolué au cours des années. Les personnes âgées sont en général très réticentes à parler de leur relation de couple, surtout si cette relation comporte des éléments abusifs. En conséquence, les intervenants pouvaient constater de la violence conjugale ou des conflits conjugaux présents depuis de nombreuses années mais n'étaient pas toujours en mesure d'identifier les éléments qui nous auraient permis de comprendre de quelle façon la situation s'était modifiée au cours des années.

b) La perte d'autonomie du partenaire qui maltraite

Nous avons vu que près de la moitié des conjoints qui maltraitent leur partenaire sont dépendants de ce dernier sur le plan physique. L'analyse des histoires de cas a par ailleurs révélé que plusieurs hommes ont de la difficulté

16. Dans notre échantillon, seulement deux femrnes âgées ont quitté leur mari violent. L'une est revenue sous les menaces de son conjoint de la tuer et de se suicider, et sous la pression de ses enfants qui considéraient qu'à son âge, sa place était auprès de son mari. L'autre s'est réfugiée dans une maison d'hébergement pour femmes violentées et n'est pas retournée avec son mari. 
à accepter leur perte d'autonomie physique. Conditionnés à être les plus forts et à contrôler leur environnement, il leur semble plus difficile d'accepter une baisse de vitalité et une dépendance vis-à-vis des autres, et en particulier de leur femme. C'est leur statut à l'intérieur du couple qui se trouve ainsi affecté. Certains conjoints deviennent alors très exigeants envers leur femme qui, de leur point de vue, n'en font jamais assez pour eux. En outre, la conjointe peut elle-même se retrouver en perte d'autonomie et donc être moins en mesure de s'occuper de son mari, ce qui augmente la frustration de ce dernier, et les tensions dans le couple. Les mauvais traitements se traduisent alors par des demandes excessives, des critiques, des insultes, et parfois même des coups. En effet, si les problèmes de santé du conjoint qui violente peuvent faire diminuer la violence physique présente depuis des années, dans d'autres cas ils peuvent l'accentuer. Quant à la violence psychologique, qui, elle, n'exige pas de force physique de la part de celui qui l'exerce, il n'y a aucune contrainte à sa continuation ou d̀ son augmentation.

À la dépendance physique vécue par le partenaire qui maltraite s'ajoute la dépendance psychologique à l'égard du conjoint que l'on maltraite. Les conjoints qui maltraitent démontrent dans une proportion de $84 \%$ de la dépendance psychologique envers leur partenaire.

La dépendance est un concept clé dans la littérature portant sur les mauvais traitements à l'égard des personnes âgées (Pillemer, 1985; Steinmetz, 1988). Nous l'avons vu, plusieurs auteurs affirment que les personnes âgées sont exposées aux mauvais traitements parce qu'elles sont dépendantes physiquement, psychologiquement ou financièrement d'une autre personne. Or, des études ont démontré que certains mauvais traitements étaient non pas dirigés vers des personnes âgées dépendantes physiquement, mais plutôt infligés par des personnes manifestant une certaine dépendance physique, psychologique ou financière envers la personne âgée (entre autres les enfants adultes aux prises avec des problèmes de toxicomanie ou de santé mentale et qui dépendent d'une manière ou d'une autre de leurs parents âgés) (Wolf et al., 1984 ; Pillemer, 1985). Nos résultats vont dans le même sens et indiquent que la perte d'autonomie et la dépendance psychologique caractérisent un grand nombre de conjoints âgés qui maltraitent.

\section{c) La perte d'autonomie du partenaire maltraité}

Dans d'autres cas, c'est l'homme qui est l'aidant et qui maltraite sa conjointe. Celle-ci, affaiblie physiquement, devient une cible encore plus vulnérable aux mauvais traitements. Comme nous l'avons déjà mentionné, il s'agit, selon plusieurs auteurs, de la situation typique : la personne âgée et vulnérable physiquement dépend d'un aidant pour ses soins quotidiens (Lau et Kosberg, 1979 ; Steinmetz et Amsden, 1983 ; Steinmetz, 1988). 
Encore une fois, la diminution des capacités physiques, cette fois-ci de la conjointe, modifie les interactions entre les partenaires. Alors que la responsabilité de veiller au bon fonctionnement de la maison et au bien-être de la famille revient traditionnellement à la femme, la perte d'autonomie de celle-ci force à une inversion des rôles entre les conjoints. C'est maintenant le mari qui doit pourvoir au bien-être de sa conjointe. Ce nouveau rôle d'aidant, auquel la plupart des hommes, surtout de cette génération, ne sont pas préparés, s'avère une source importante de stress. Nul doute qu'une telle situation, lorsqu'en plus les relations entre les partenaires sont peu harmonieuses, augmente la vulnérabilité de la conjointe aux mauvais traitements.

Chez les couples lucides, nous avons dénombré sept femmes parmi les 32 conjoints auteurs de mauvais traitements. Parmi elles, quatre sont aussi maltraitées par leur conjoint. En dépit du petit nombre de situations où des femmes âgées étaient auteures de mauvais traitements, deux constatations ont pu être dégagées. Premièrement, lorsque les deux conjoints s'infligent mutuellement des mauvais traitements, le plus souvent les comportements abusifs s'inscrivent dans un contexte où les deux conjoints sont surtout violents psychologiquement l'un envers l'autre, mais parfois aussi physiquement, depuis des années. Deuxièmement, on remarque que les mauvais traitements infligés par les femmes peuvent être exacerbés par la perte d'autonomie du mari ou apparaître avec celle-ci. Cette dernière constatation soulève une interrogation : se pourrait-il que certaines femmes, ayant subi de la violence de la part de leur conjoint pendant une grande partie de leur vie conjugale, s'approprient un nouveau pouvoir maintenant que celui-ci est en perte d'autonomie et le maltraitent en réaction à leur passé conjugal ?

\section{LES SITUATIONS DE MAUVAIS TRAITEMENTS LORSQUE L'UN DES CONJOINTS SOUFFRE DE PERTES COGNITIVES}

Dans 29 situations impliquant un couple âgé, l'un des conjoints souffre de pertes cognitives. Ce conjoint peut être victime des mauvais traitcments (7) ou auteur des mauvais traitements (8), ou encore être les deux à la fois, lorsqu'il y a mauvais traitements réciproques $\left(7^{17}\right)$.

Aux fins de l'analyse, lorsqu'il y a mauvais traitements mutuels, les deux conjoints se retrouvent à la fois parmi ceux qui maltraitent et parmi ceux qui sont maltraités. Ce qui signifie que l'analyse porte sur 14 situations où le partenaire ayant des pertes cognitives est maltraité et 15 situations où il est celui qui maltraite.

17. Ces sept dossiers comptent aux fins de l'analyse pour 14 situations de mauvais traitements puisqu'on identifie deux victimes par dossier. 


\subsection{Le conjoint qui maltraite souffre de pertes cognitives}

Le plus souvent (13/15), le conjoint ayant des pertes cognitives et qui maltraite est le mari. Les pertes cognitives étant associées à un âge avancé, les conjoints qui en souffrent sont les plus âgés parmi ceux qui maltraitent. Près de sept sur dix ont 80 ans et plus. Vu leur grand âge et leurs pertes cognitives, seulement deux sur 15 sont completement autonomes pour les activités de la vie quotidienne. Les victimes, elles aussi, sont assez âgées. Elles ont, en moyenne, 76,2 ans. Toutefois, malgré leur âge respectable, la majorité (13/15) sont complètement autonomes pour les activités de la vie quotidienne, et 12 d'entre elles agissent en tant qu'aidantes auprès de leur partenaire. Les mauvais traitements infligés par ces conjoints en perte de lucidité sont essentiellement de nature psychologique (15/15) et - moins fréquemment - de nature physique (5/15).

Certains mauvais traitements s'inscrivent encore une fois dans une problématique de violence conjugale déjà présente depuis plusieurs années et qui se perpétue avec les pertes cognitives de l'un des conjoints. Dans d'autres cas, les mauvais traitements semblent être la conséquence directe des pertes cognitives du partenaire. Ils apparaissent avec l'aggravation des problèmes cognitifs. On sait tout particulièrement que la maladie d'Alzheimer et les accidents cérébro-vasculaires ont pour effet dans bien des cas de modifier le comportement du patient. D'après des recherches américaines, on estime que plus de la moitié des personnes atteintes de démence manifestent certaines formes de comportements agressifs tels que la violence verbale et physique (Ryden, 1988 ; Hamel et al., 1990).

\subsection{Le conjoint maltraité souffre de pertes cognitives}

On retrouve plus de conjoints (8) que de conjointes (6) victimes de mauvais traitements et souffrant de pertes cognitives. Ces conjoints, d'une moyenne d'âge de 80,9 ans, sont les plus âgés parmi les conjoints victimes. Aucun d'eux n'est totalement autonome pour les activités de la vie quotidienne. À l'opposé, les conjoints qui maltraitent sont tous autonomes et sont tous les aidants de leur partenaire.

Les conjoints aidants, le plus souvent, négligent leur partenaire (9/14) ou font usage de violence psychologique envers eux (7/14). Enfin, dans seulement deux cas sur 14, les aidants ont été violents physiquement. Encore une fois, précisons que dans la moitié de ces situations, on observe des mauvais traitements réciproques. Le partenaire atteint de pertes cognitives est violent psychologiquement et parfois physiquement.

Ce qui émerge de l'analyse, c'est la méconnaissance, de la part des conjoints aidants, des impacts des troubles cognitifs dont souffrent leur 
partenaire. En effet, les conjoints aidants ne semblent pas bien saisir les effets de ces pertes de lucidité sur le comportement de leur partenaire. En outre, ils comprennent mal les nouveaux besoins qu'une telle situation engendre. Plus spécifiquement, les aidants ne semblent pas réaliser à quel point il est risqué de laisser livrées à elles-mêmes les personnes qui souffrent de pertes cognitives, et le fait que les comportements agressifs des personnes atteintes de pertes cognitives et en particulier de la maladie d'Alzheimer, sont une des composantes de l'évolution de la maladie.

La méconnaissance des troubles cognitifs dont souffrent leur partenaire amène les conjoints aidants à croire que ceux-ci sont en meilleure condition physique et mentale qu'ils ne le sont en réalité, et qu'ils peuvent en grande partie s'occuper d'eux-mêmes. Cette fausse perception de la réalité conduit les aidants à négliger leur partenaire en le laissant seul, sans surveillance adéquate, ou à être agressif verbalement et parfois physiquement envers lui, en lui demandant de faire des choses dont il est incapable.

Ce manque d'information au sujet des effets des troubles cognitifs sur ceux qui en souffrent paraît constituer un élément important dans la compréhension de certaines situations de mauvais traitements. Toutefois, d'autres facteurs peuvent évidemment nous aider à comprendre ces situations. La problématique d'une situation de violence conjugale récurrente est également présente ici, bien que beaucoup moins souvent que lorsque les deux conjoints sont lucides. En outre, le stress de l'aidant qui doit s'occuper de son conjoint atteint de démence constitue également un élément qui peut favoriser les mauvais traitements. Il est reconnu que la tâche consistant à prendre soin d'une personne souffrant de la maladie d'Alzheimer est très stressante, d'autant plus lorsque l'aidant, bien qu'autonome physiquement, est assez âgé, et connaît lui-même certains ennuis de santé.

\subsection{Les mauvais traitements réciproques}

Nous avons vu que dans près du tiers des situations où l'un des conjoints souffre de pertes cognitives, il y a mauvais traitements réciproques entre les partenaires. Lorsqu'il y a des mauvais traitements mutuels, on retrouve une combinaison des deux types de situations décrites précédemment. D'une part, le partenaire qui est atteint de troubles cognitifs et en particulier de la maladie d'Alzheimer, voit son comportement se modifier sous l'effet de la maladie : la personne devient plus demandante et plus agressive verbalement, parfois physiquement. Ce comportement agressif peut être le prolongement d'une relation de couple où le conjoint faisait déjà usage de violence physique et psychologique, ou il peut apparaître progressivement dans une relation exempte de violence. D'autre part, tantôt en réaction à ce comportement agressif, tantôt par méconnaissance de la maladie, tantôt en raison du stress 
que génère sa tâche d'aidant, le conjoint aidant néglige le conjoint souffrant de pertes cognitives ou lui fait subir des mauvais traitements psychologiques ou physiques.

\section{CONCLUSION}

L'étude dont est tiré cet article visait une meilleure compréhension des situations de mauvais traitements à l'égard des personnes âgées et des dynamiques qui les sous-tendent, de manière à mieux intervenir dans de telles situations. Pour ce faire, nous avons décidé d'examiner les mauvais traitements sous l'angle de problématiques spécifiques. Nous avons utilisé comme premier critère de distinction les liens qui unissent la personne âgée et l'auteur des mauvais traitements. D'autres critères de distinction se sont ajoutés, telles les pertes cognitives dont souffre l'un des conjoints, s'inspirant de la spécificité de certaines situations de mauvais traitements.

En explorant davantage ces problématiques spécifiques, nous pouvons aller au-delà de la compréhension actuelle du problème des mauvais traitements à l'égard des personnes âgées qui, jusqu'à présent, s'est souvent limitée à une seule de ces facettes : les mauvais traitements infligés par un aidant qui doit s'occuper d'un parent âgé en perte d'autonomie. Nous pouvons dépasser les lieux communs qu'engendre une telle façon d'appréhender le problème : par exemple, nos données indiquent, entre autres, que les conjoints âgés maltraités ne sont pas tous en perte d'autonomie et dépendant physiquement d'un aidant, que les conjoints qui maltraitent sont aussi nombreux que les conjoints maltraités à ne pas être entièrement autonomes sur le plan physique, que les aidants ne sont pas que des auteurs de mauvais traitements potentiels, qu'ils peuvent aussi, $s$ 'ils sont âgés, être maltraités par la personne âgée dont ils ont la charge.

Concrètement, de tels résultats remettent en question la validité des outils de dépistage qui ont été développés au cours des dernières années. Ces outils de dépistage des mauvais traitements sont construits à partir de facteurs de risques inférés des caractéristiques des victimes et des auteurs de mauvais traitements provenant des études sur le sujet. Entre autres, la plupart de ces outils accordent une grande importance à des éléments tels que la perte d'autonomie physique et mentale de la personne âgée et le stress de l'aidant. Or, il semble évident que de tels outils ne permettent pas d'identifier l'ensemble des situations de mauvais traitements mais une partie seulement de celles-ci.

En ce qui a trait plus spécifiquement aux mauvais traitements entre conjoints âgés qui s'inscrivent dans un contexte de violence conjugale présent depuis plusieurs années, la question des interventions possibles pour aider 
ces femmes se pose. De quelle manière peut-on intervenir ? Étant donné leur âge et leurs valeurs, faut-il intervenir auprès d'elles comme on le fait auprès de leurs consœurs plus jeunes ? Il s'agit d'une question cruciale qui donne matière à réflexion à tous ceux et celles qui cuvrent auprès des femmes victimes de violence conjugale et celle des personnes âgées victimes de mauvais traitements.

Enfin, en ce qui a trait aux mauvais traitements impliquant un conjoint souffrant de pertes cognitives, nos résultats montrent que la méconnaissance, de la part du conjoint aidant, des impacts des pertes cognitives sur le comportement de celui qui en souffre, constitue un élément important dans la compréhension de ces situations de mauvais traitements. En conséquence, nous souhaitons que les programmes d'information visant les familles de personnes atteintes de pertes cognitives et plus spécifiquement de la maladie d'Alzheimer, déjà implantés un peu partout au Québec, soient davantage publicisés, afin d'atteindre un plus grand nombre de familles. Une meilleure compréhension des composantes de la maladie par les familles ainsi qu'un meilleur soutien de celles-ci devraient contribuer à prévenir plusieurs situations abusives.

\section{BIBLIOGRAPHIE}

ARONSON, J., THORNEWELL, C. et WILLIAMS, K. (1995), « Wife Assault in Old Age : Corning out of Obscurity ", Aging/Vieillissement. La Revue canadienne du veillissement/ Canadian Journal of Aging, 14, (hors-série/supplément 2), 72-88.

BLOCK, M. R. et SINNOTT, J. D. (1979), The Battered Elder Syndrome : An Exploratory Study, College Park, MD : University of Maryland, Center of Aging.

FINKELHOR, D. et PILLEMER, K. (1988), « Elder abuse : Its relationship to other forms of domestic violence ", dans G. T. HOTALING, D. FINKELHOR, J. T. KIRKPATRICK et M. A. STRAUS (éd.), Family Abuse and Its Consequences : New Directions in Research, Sage Publications : 244-254.

GESINO, I. p., SMITH, H. H. et KECKICH, w. A. (1982), « The battered woman grows old ", Clinical Gerontologist, 1, (1), 50-67.

GIORDANO, N. H. (1982), Individual and Family Correlates of Elder Abuse, Doctoral Dissertation, University of Georgia.

GIORDANO, N. H. et GIORDANO J. A. (1984), « Elder abuse. A review of the literature ", Social Work, 29, (3), 232-236.

GRANDMAISON, A. (1988), Protection des personnes âgées : Etude exploratoire de la violence à l'égard de la clientèle des personnes âgées du CSSMM, Montréal : Centre des services sociaux du Montréal métropolitain. Service de la recherche et des normes de pratiques.

HAMEL, M., GOLD, D. P., ANDRES, D., REIS, M., DASTOOR, D., GRAUER, H. et BERGMAN, H. (1990), "Predictors and consequences of aggressive behavior by community-based dementia patients ", The Gerontologist, 30, 206-211. 
HARRIS, S. B. (1996), « For better or for worse : spouse abuse grown old ", Joumal of Abuse and Neglect, 8, (1), 1.33.

LAU, E. et KOSBERG, J. (1979), « Abuse of the elderly by informal care providers ", Aging, September-october, 10-15.

LÉVESQUE, M.-J. (1990), « Les personnes âgées maltraitées. Éléments de problématique et bibliographie sélective (1980-1987) », dans J. CARETTE et L. PLAMONDON, Vieillir sans violence, Presses de l'Université du Québec : 29-58.

MACLEAN, M. J. (éd.). (1995), Abuse and Neglect of Older Canadians : Srategies for Changes, Toronto : Thompson Educational Publishing.

McDONALD, P. L., HORWICK, J. P., ROBERTSON, G. B. et WALLACE, J. E. (1991), Elder Abuse and Neglect in Canada, Toronto : Butterworths.

NAMIASH, D. (1990), « L'intervention en situation de risque d'abus en milieu naturel ", dans J. CARETTE et L. PLAMONDON, Vieillir sans violence, Presses de l'Université du Québec : $87-95$.

PAGELOW, M. D. (1981), Woman-Battering : Victims and Their Experiences, Beverly Hills : Sage.

PILLEMER, K. (1985), " The dangers of dependency : new findings on domestic violence against the elderly », Social Problems, 33, 146-158.

PILLEMER, K. et FINKELHOR, D. (1988), « The prevalence of elder abuse : a random sample survey ", The Gerontological Society of America, 8, (1), $51-57$.

PODNIEKS, E. et PILLEMER, K. (1990), L'étude Ryerson : Une enquête nationale sur les mauvais traitements des personnes âgées au Canada, Toronto : Ryerson Polytechnical Institute.

ROY, M. (1982), The Abusive Partner : An Analysis of Domestic Battering, Toronto : Ontario Ministry of Community and Social Services.

RYDEN, M. (1988), "Aggressive behavior in persons with dementia living in the community ", The Alzheimer Disease and Associated Disorder International Journal, 2, 342-355.

SHELL, D. J. (1982), Protection of the Elderly. A study of Elder Abuse, Manitoba Council on Aging, Winnipeg, Manitoba : Manitoba Association on Gerontology.

STEINMETZ, S. (1988), Duty Bound : Elder Abuse and Family Care, Newbury Park : Sage Publications.

STEINMETZ, S. et AMSDEN, D. J. (1983), « Dependent elders, family stress and abuse ", dans T. H. Brubaker (éd.), Family Relationships in Later Life, Berverly Hills, CA : Sage : 173-192.

SUITOR, J. J., PILLEMER, K. A. et STRAUS, M. A. (1990), "Marital violence in a life course perspective », dans M. A. STRAUS et R. GELLES, Physical Violence in American Families: Risk Factors and Adaptations to Violence in 8145 Families, New Brunswick, NJ : Transaction Publishers.

VALENTINE, D. et CASH, T. (1986), "A definitional discussion of elder maltreatment ", Journal of Gerontological Social Work, 9, (3), 17-28.

VINTON, L. (1992), « Battered women's shelters and older women : the Florida experience ", Journal of Family Violence, 7, (1), 63-72.

WOLF, R., GODKIN, M. et PLLEMER, K. (1984), Elder Abuse and Neglect : Report from Three Model Projects, Worcester, MA : University of Massachusetts Medical center. 